\title{
ESCOAMENTO DE FERRO-GUSA EM CANAL DE ALTO- FORNO E SUA RELAÇÃO COM O DESGASTE DO REVESTIMENTO REFRATÁRIO
}

\author{
R. V. P. REZENDE ${ }^{1}$, A. A. ULSON DE SOUZA ${ }^{1}$, S. M. A. GUELLI ULSON DE SOUZA ${ }^{1}$, \\ A. F. C. SILVA ${ }^{2}$, C. R. MALISKA ${ }^{2}$ \\ ${ }^{1}$ Universidade Federal de Santa Catarina, Departamento de Engenharia Química e \\ Engenharia de Alimentos. \\ ${ }^{2}$ Universidade Federal de Santa Catarina, Departamento de Engenharia Mecânica. \\ E-mail para contato: rezendervp@gmail.com
}

\begin{abstract}
RESUMO - Historicamente, os processos siderúrgicos estão vinculados ao desenvolvimento técnico e industrial de uma nação e estão atrelados à quantidade e à qualidade do aço produzido. Um dos equipamentos importantes neste processo, o canal de alto-forno, cuja função principal é extravasar o metal do altoforno, sofre desgaste de seu revestimento refratário - parte fundamental de sua durabilidade - e pouco se sabe como este processo ocorre. A metodologia apresentada neste trabalho modela o sistema ar-gusa considerando tanto o jato aberto quanto a superfície livre, além da sua natureza transiente e turbulenta. O simulador ANSYS CFX foi empregado na solução do sistema de equações. Os resultados indicam que o caráter vorticoso e oscilatório na região de impacto do jato aumentam os níveis energia cinética turbulenta, a qual apresenta correlação com a taxa de desgaste, permitindo estimar o seu perfil, e contradizem a crença de que as tensões cisalhantes são as principais responsáveis por este processo.
\end{abstract}

\section{INTRODUÇÃO}

O canal de alto-forno consiste em uma calha de cerca de $20 \mathrm{~m}$ de comprimento e pouco mais de $1 \mathrm{~m}$ de largura e profundidade. Sua função consiste em direcionar o metal fundido do alto-forno para os carros-torpedos (vagões de transporte) separando o ferro-gusa da escória, além de homogeneizar a temperatura do metal fundido. $O$ metal então é transportado até operações de refino que variam de acordo com a finalidade do produto final. A qualidade desta operação unitária determina o tempo e rendimento das operações subsequentes.

Ao fim do canal espera-se que o ferro-gusa e a escória estejam separados por flotação e que as temperaturas estejam mais homogêneas. No caso analisado, especificamente, a temperatura de operação é cerca de $1650{ }^{\circ} \mathrm{C}$ com vazões mássicas que variam de $5-14$ ton $/ \mathrm{min}$ (mas isto depende de cada planta operacional). O extravasamento do alto-forno é feito por meio de um orifício na parede do mesmo resultando em um jato aberto de uma mistura bifásica de metal e escória fundidos com uma velocidade de saída de 7,5m/s em um ângulo de $10^{\circ}$, o qual colide com a escória sobrenadante ao ferro-gusa fundido a $3,5 \mathrm{~m}$ da parede do altoforno. A operação leva cerca de $1,5 \mathrm{~h}$ sendo revezada em diversos canais em torno do alto 
forno. Um canal tem um tempo de vida de cerca de um mês.

O tempo de vida de cada canal é determinado pelo desgaste do revestimento refratário, sendo as regiões mais críticas as interfaces metal-escória, e a zona de impacto do jato; ambas com um desgaste acentuado nas laterais. O fundo do canal é levemente atacado e apresenta um leve abaulamento apenas na região de impacto do jato. As condições de operação extremas dificultam uma reprodução experimental consoante com a realidade industrial, tornado difícil o estudo deste processo.

Alguns trabalhos estudaram esta dinâmica por modelos físicos e métodos numéricos (Begnis, et al., 2005; He, Evans, et al., 2002; He, Zulli, et al., 2002; Kim, et al., 1998; Luomala, et al., 2001; Shestopalov, et al., 1988; Stevenson e He, 2005). Contudo, ou a similaridade dimensional era comprometida por emulsificação das fases; ou os modelos matemáticos e tratamentos numéricos não levaram em consideração a dinâmica do jato e da superfície livre. O jato é desconsiderado e seu ponto de impacto tratado como uma região de entrada com velocidade e ângulo prescritos; e a superfície como uma parede com condição de escorregamento. Apesar das contribuições destes trabalhos, nenhum foi capaz de predizer ou mesmo indicar uma correlação entre a fluidodinâmica e a taxa de desgaste.

E, apesar da simplicidade e ganhos de tempo computacional, estas simplificações geram um inconveniente: campos simétricos de linhas de corrente e um escoamento - quase sempre monofásico - comportando-se como um escoamento interno ao invés de um escoamento em canal aberto. Foi demonstrado que a iteração entre o jato aberto e a superfície livre é a que melhor descreve a dinâmica do escoamento, sendo capaz de capturar oscilações, propagações de ondas e o entranhamento de ar no metal fluido. Uma abordagem deste tipo é multifásica, transiente e turbulenta (Rezende, 2008).

Neste trabalho, estas características são consideradas, e uma correlação entre o escoamento e a taxa de desgaste é proposta. O escoamento é considerado bifásico formado por ferro-gusa fundido e ar. Nem a escória e nem a transferência de calor são consideradas por simplicidade. O modelo matemático foi resolvido numericamente no simulador comercial ANSYS CFX $11.0^{\circ}$.

\section{MODELO MATEMÁTICO}

O modelo consiste na equação de conservação de massa e volume, respectivamente,

$$
\begin{aligned}
& \frac{\partial}{\partial t}\left(r_{\alpha}\left\langle\rho_{\alpha}\right\rangle\right)+\nabla \cdot\left(r_{\alpha}\left\langle\rho_{\alpha}\right\rangle\langle\mathbf{u}\rangle\right)=0, \\
& \sum_{\alpha=1}^{2} r_{\alpha}=1
\end{aligned}
$$

conservação da quantidade de movimento, considerando o modelo homogêneo, tensão superficial e comportamento reológico newtoniano: 


$$
\frac{\partial}{\partial t}(\langle\rho\rangle\langle\mathbf{u}\rangle)+\nabla \cdot(\langle\rho\rangle\langle\mathbf{u}\rangle \otimes\langle\mathbf{u}\rangle)=\nabla \cdot\left(\mu_{e f f}\left\langle\nabla \mathbf{u}+\nabla \mathbf{u}^{T}\right\rangle\right)-\nabla\langle p\rangle+\left(\langle\rho\rangle-\rho_{r e f}\right) \mathbf{g}+\mathbf{m}_{i}^{\sigma}
$$

A tensão superficial $\mathbf{m}_{i}^{\sigma}$ é formulada de acordo com o modelo de Brackbill, et al. (1992), e o tratamento URANS para turbulência usando o modelo a duas equações $k-\varepsilon$,

$$
\begin{aligned}
& \frac{\partial(\langle\rho\rangle\langle k\rangle)}{\partial t}+\nabla \cdot(\langle\rho\rangle\langle\mathbf{u}\rangle\langle k\rangle)=\nabla \cdot\left[\left(\mu+\frac{\mu_{T}}{\sigma_{k}}\right) \nabla\langle k\rangle\right]+\ldots \\
& \ldots+\left(\mu_{T}\left\langle\nabla \mathbf{u}+\nabla \mathbf{u}^{T}\right\rangle: \nabla\langle\mathbf{u}\rangle-\langle\rho\rangle\langle\varepsilon\rangle\right) \\
& \frac{\partial}{\partial t}(\langle\rho\rangle\langle\varepsilon\rangle)+\nabla \cdot(\langle\rho\rangle\langle\mathbf{u}\rangle\langle\varepsilon\rangle)=\nabla \cdot\left[\left(\mu+\frac{\mu_{T}}{\sigma_{\varepsilon}}\right) \nabla\langle\varepsilon\rangle\right]+\ldots \\
& \ldots+\frac{\langle\varepsilon\rangle}{\langle k\rangle}\left(C_{\varepsilon 1} \mu_{T}\left\langle\nabla \mathbf{u}+\nabla \mathbf{u}^{T}\right\rangle: \nabla\langle\mathbf{u}\rangle-C_{\varepsilon 2}\langle\rho\rangle\langle\varepsilon\rangle\right),
\end{aligned}
$$

onde a viscosidade turbulenta é definida como

$$
\mu_{T}=C_{\mu} \rho \frac{k^{2}}{\varepsilon} .
$$

As condições de contorno que completam o modelo são indicadas na Tabela 1.

Tabela 1 - Condições de contorno empregadas no trabalho.

\section{Local}

Tap hole

Refractory wall

Saída

Cast-iron outlet

Far field
Tipo de Condição

Entrada

Abertura com Pressão e direção normal especificada

Abertura com Pressão estática especificada

\section{Condição de Contorno}

$7.5 \mathrm{~m} / \mathrm{s}$ com $10^{\circ}$ de inclinação

No slip para u e lei de parede para $k-\varepsilon$

$$
p=0 P a \quad r_{\text {air }}=1 \quad I_{\text {turb }}=1 \%
$$

$$
p=0 \mathrm{~Pa} \quad r_{\text {air }}=1 \quad I_{\text {turb }}=10 \%
$$

O domínio de cálculo é apresentado na Figura 1. Nela, as regiões da Tabela 1 são indicadas. A malha é formada por aproximadamente 400 mil elementos hexaédricos. As condições iniciais são de fluido quiescente no interior do canal, e as frações volumétricas são inicializadas de acordo com 


$$
\begin{aligned}
& r_{\text {gusa }}^{o}=\left(0.5 \cdot \tanh \left(\frac{h^{o}-y}{\delta_{i}}\right)+0.5\right), \\
& r_{\text {ar }}^{o}=1-r_{\text {gusa }}^{o} .
\end{aligned}
$$

A operação real leva entre 1,5 a $2 \mathrm{~h}$, o que com um passo de tempo de cerca de $0,001 \mathrm{~s}$ torna a análise numérica transiente proibitiva. A forma encontrada para contornar esta dificuldade é mostrada na Figura 2. Após 35s de simulação física, o suficiente para que o sistema alcance um regime oscilatório harmônico, o último passo de tempo é empregado em uma simulação em regime permanente que ainda mantém o termo transiente, sem, contudo, convergir a cada passo de tempo. Esta liberdade permite o uso de passos de tempo de até $5 \mathrm{~s}$. Com a proximidade do tempo físico de $2 \mathrm{~h}$, o passo de tempo e os critérios de convergência iniciais $\left(\mathrm{RMS}<10^{-5}\right.$ ) foram novamente reestabelecidos a fim de garantir a precisão dos resultados.

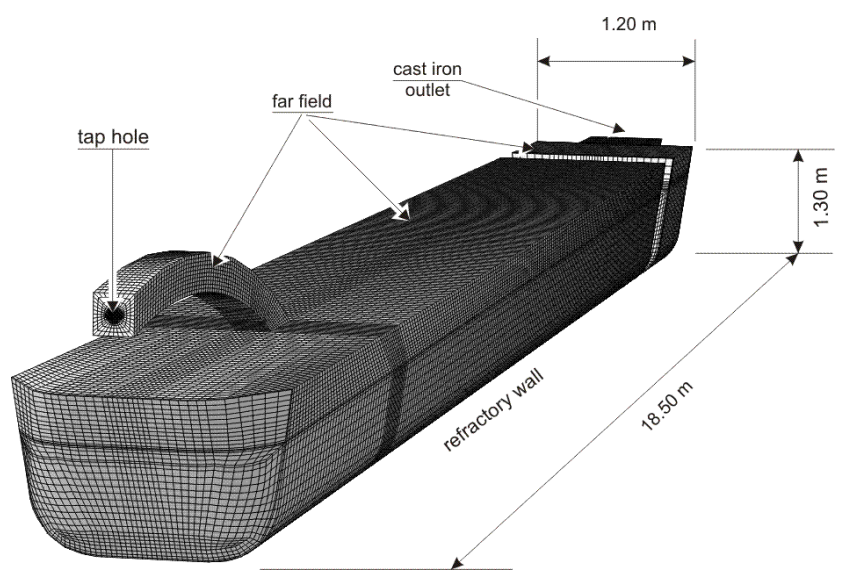

Figura 1 - Malha e regiões do domínio onde as condições de contorno são atribuídas.

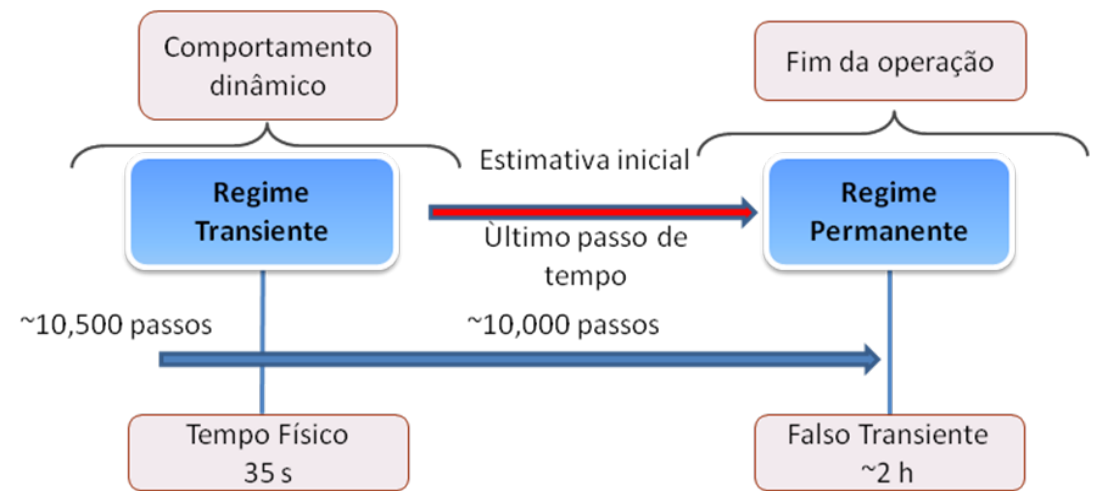

Figura 2 - Abordagem numérica empregada para se alcançar a escala de tempo da operação real.

As propriedades físicas são listadas da Tabela 2. 
Tabela 2 - Propriedades Físicas

\begin{tabular}{|c|c|c|c|c|}
\hline$\rho_{\text {gusa }}$ & $\rho_{a r}$ & $\mu_{\text {gusa }}$ & $\mu_{a r}$ & $\sigma_{\alpha \beta}$ \\
\hline $7000 \mathrm{~kg} / \mathrm{m}^{3}$ & $1,185 \mathrm{~kg} / \mathrm{m}^{3}$ & $5 \times 10^{-3}$ Pa.s & $1,83 \times 10^{-5}$ Pa.s & $1,35 \mathrm{~N} / \mathrm{m}$ \\
\hline
\end{tabular}

\section{RESULTADOS E DISCUSSÃO}

Na Figura 3 comparam-se os resultados obtidos neste trabalho com o modelo físico em escala de água e óleo de Begnis et al. (2005). Ambos os resultados apresentam boa similaridade. O entranhamento de ar também é evidente no modelo numérico e no físico, o qual apresenta bolhas eclodindo à frente da região de impacto do jato. Este fenômeno não é possível de ser capturado sem se considerar a colisão do jato com a superfície livre.

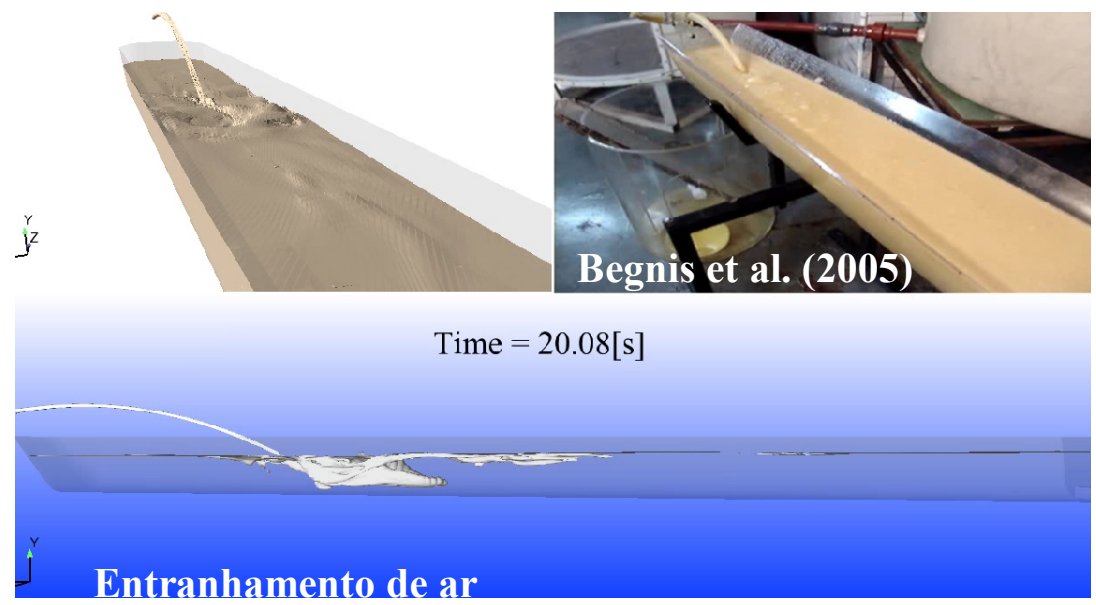

Figura 3 - Comparação entre o modelo numérico deste trabalho e o modelo físico de Begnis et al. (2005).

O comportamento oscilatório harmônico também é bem descrito pelo modelo. Na Figura 4 são mostrados o campo de tensão cisalhante na parede do refratário e o entranhamento do ar nas isossuperfícies de fração volumétrica em cinza. Os picos de tensão oscilam transversalmente à direção do escoamento com valores de mais de $80 \mathrm{~Pa}$, enquanto nas laterais se mantém em torno de $20 \mathrm{~Pa}$. Contudo, o fundo do canal apresenta um desgaste pouco significativo enquanto que nas laterais, principalmente junto às interfaces, este perfil é muito acentuado, indicando que, isoladamente, a tensão cisalhante não pode ser a responsável pelo perfil de desgaste observado na prática.

Dois fatores concorrem nestas regiões: a presença de ar, e a intensidade turbulenta. Praticamente não há ar tocando o fundo do canal enquanto nas laterais e interface ocorre uma mistura consideravel, principalemente na região de impacto do jato. Já a tubulência mais intensa aprimora esta mistura entre as fases. É sabido que ocorrem diversas reações químicas entre o refratário, metal, o ar,e a escória (Mukai, 1998; Mukai, et al., 1989), e a presença de ar e a mistura aprimorada pelas flutuações de velocidade turbulenta, associadas a uma tensão cisalhante oscilante podem então promover a corrosão, dissolução e abrasão do refratário.

A Figura 5 compara o perfil real de desgaste com o perfil de energia cinética turbulenta. 
Os perfis são praticamente os mesmos e apresentam um índice de correlação de $97 \%$. Todavia, o desgaste não ocorre onde somente a energia cinética turbulenta é mais intensa, é necessário para isto a presença de ar para que os processos químicos que permitem a dissolução e a fragilização do refratário tomem parte. Logo, a ação da dispersão de massa ocasionada pela turbulência deve ser considerada somente na linha da interface.
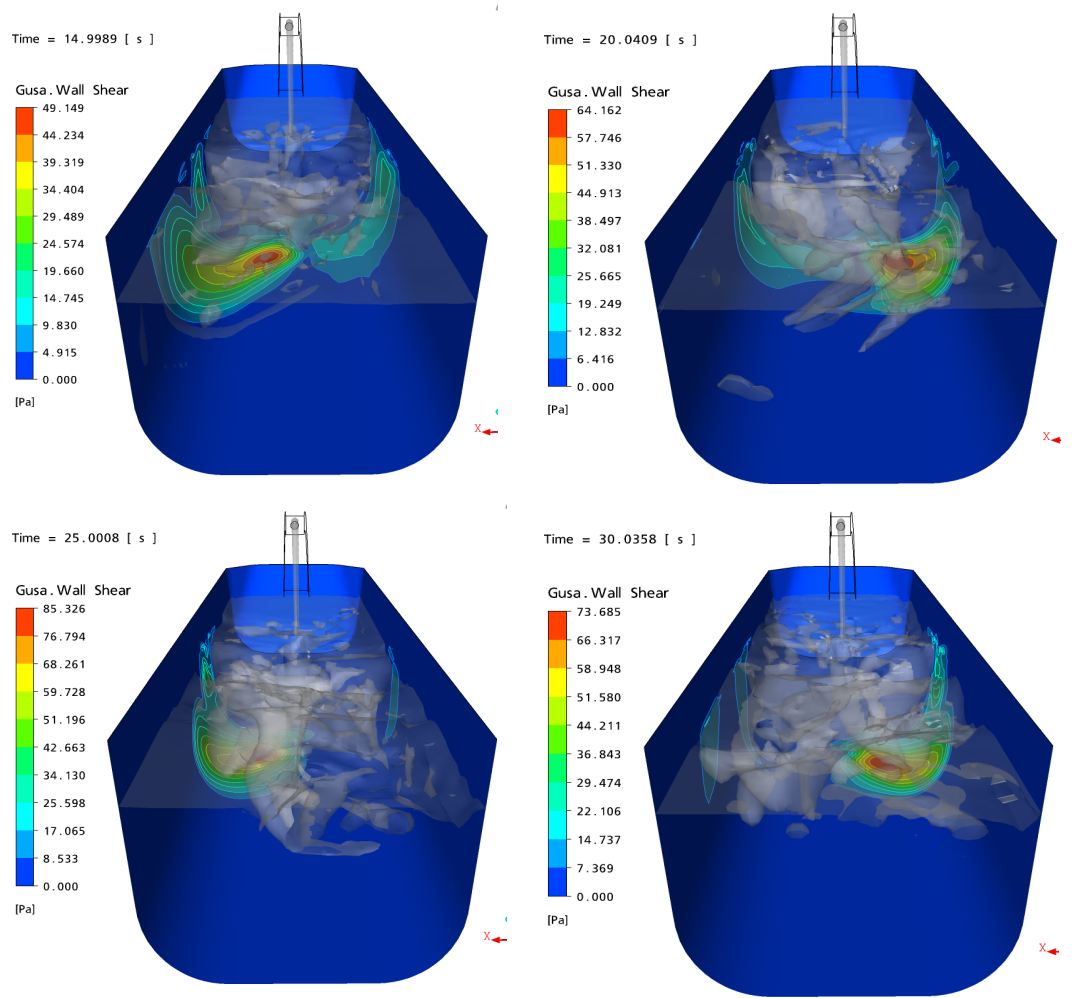

Figura 4 - Campo oscilatório de tensão cisalhante na parede do refratário e isossuperfícies de fração volumétrica de ar

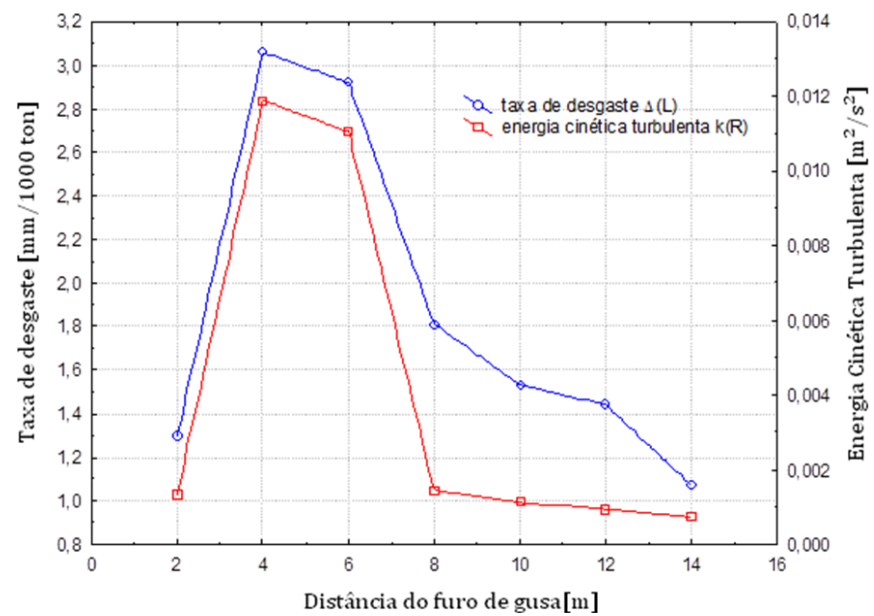

Figura 5 - Gráfico comparativo entre os perfis de desgaste obtidos de um canal real, e a energia cinética turbulenta medida nas laterais a $5 \mathrm{~cm}$ abaixo da interface.

Com base nos resultados, e considerando uma distribuição gaussiana em torno da interface, 
a taxa de desgaste média pode ser expressa por

$$
\Delta=\left(1074.107 k_{\text {gusa }}^{0.27161}\right) \cdot \exp \left[-\frac{\left(r_{\text {gusa }}-0.5\right)^{2}}{0.04}\right] \text {. }
$$

O emprego desta correlação permite a construção de uma superfície elevada resultando em uma geometria que representa a profundidade do desgaste. Na Figura 6 apresenta-se a comparação entre a geometria predita e a geometria medida in loco (amostrada a cada $2 \mathrm{~m}$ do canal). Os perfis apresentam boa concordância e a Equação 10 permite a predição da profundidade do desgaste a partir de informações do escoamento.

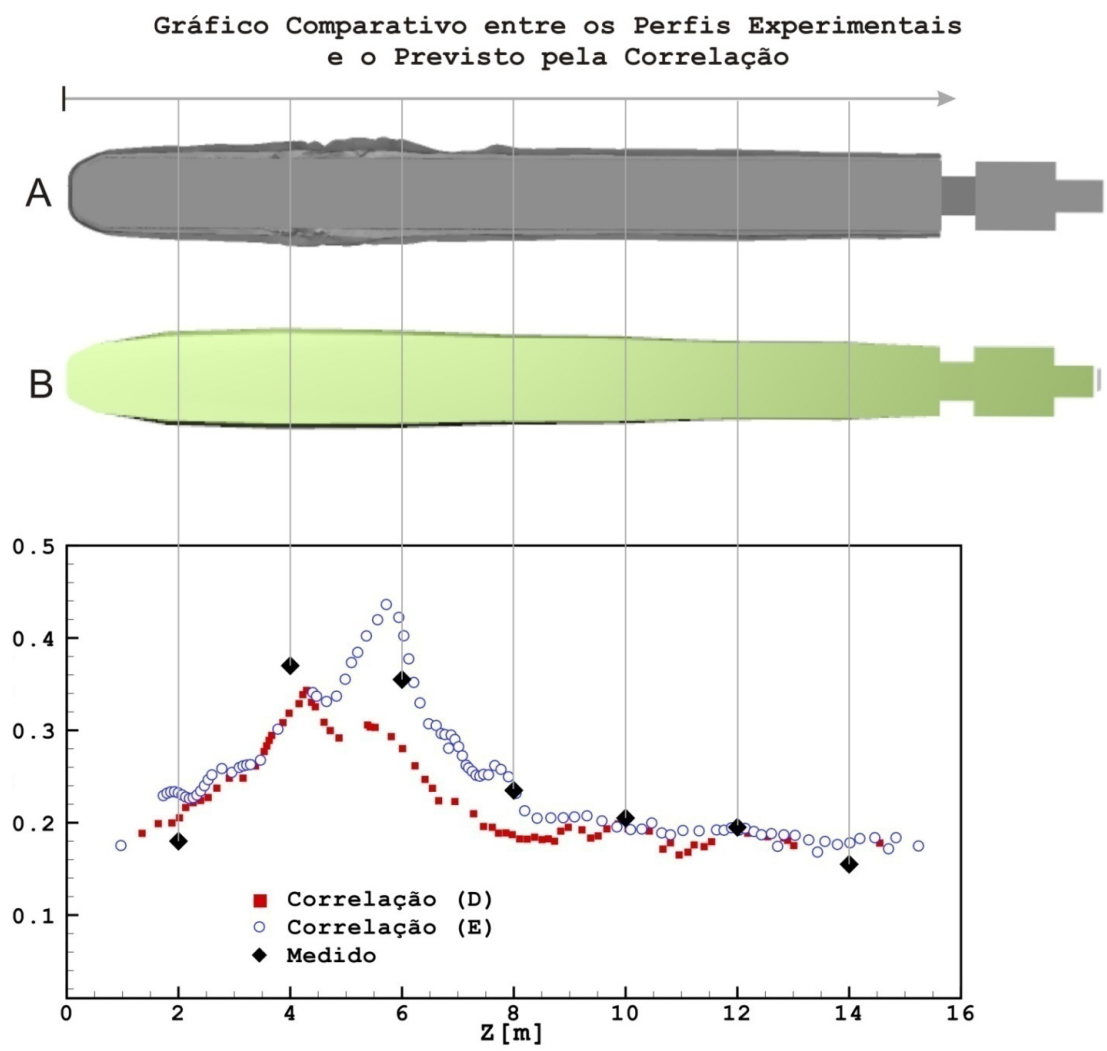

Figura 6 - Emprego da Equação 10 para predição do desgaste. Em (A) a geometria predita, e em (B) o perfil final do canal ao fim da campanha.

\section{CONCLUSÕES}

O escoamento bifásico de ar e ferro-gusa no canal de extravasamento de um alto forno foi matematicamente modelado e resolvido numericamente. Os resultados demonstraram uma boa concordância quando comparados a outros trabalhos da literatura.

Os resultados obtidos demonstraram que a abordagem multifásica é mais adequada do que a abordagem monofásica em regime permanente por descrever a interação jato/superfície livre. Ela permite capturar características físicas mais próximas da realidade 
do escoamento.

A energia cinética turbulenta e a dinâmica da interface, o que afeta a fração volumétrica de ar - fator necessário para que ocorra um ataque químico ao refratário - junto às paredes na zona de mistura, parecem estar relacionados ao desgaste. Com base nisto, uma correlação foi proposta como função da energia cinética turbulenta - variável preponderante na taxa de mistura entre o ar e o metal fundido.

A geometria final foi predita com relativo sucesso apresentando boa concordância com a geometria medida in loco pela correlação proposta. 0 cisalhamento parece ser um fator secundário. 0 modelo permite assim, estudar novas propostas de projeto e avaliar o ganho de desempenho no tempo de campanha do canal.

\section{REFERENCIAS}

BEGNIS, J. S. S., BRANDALEZE, E., \& TOPOLEVSKY, R. Simulación del canal del alto forno $n^{\circ} 2$ por medio de modelos físicos. Paper presented at the $5^{\mathrm{a}}$ Conferencia de Redución del IAS, Argentina, 2005, Noviembre.

BRACKBILL, J. U., KOTHE, D. B., \& ZEMACH, C., A continuum method for modeling surface tension. Journal of Computational Physics, v. 100. (2), p. 335-354, 1992.

HE, Q., EVANS, G., ZULLI, P., TANZIL, F., \& LEE, B., Flow characteristics in a blast furnace trough. ISIJ International, v. 42. (8), p. 844-851, 2002.

HE, Q., ZULLI, P., TANZIL, F., LEE, B., DUNNING, J., \& EVANS, G., Flow characteristics of a blast furnace taphole stream and its effects on trough refractory wear. ISIJ International, v. 42. (3), p. 235-242, 2002.

KIM, H., OZTURK, B., \& FRUEHAN, R. J., Slag-metal separation in the blast furnace trough. ISIJ International, v. 38. (5), p. 430-439, 1998.

LUOMALA, M. J., PAANEMEN, T. T., KÖYKKÄ, M. J., FABRITIUS, T. M. J., NEVALA, H., \& HÄKKI, J. J., Modelling of fluid in the blast furnace trough. Stell Research, v. 72. (4), p. 130-135, 2001.

MUKAI, K., Marangoni flows and corrosion of refractory walls. Philosophical Transactions of The Royal Society A: Mathematical, Physical and Engineering Sciences, v. 366. (1739), p. 1015-1026, 1998.

MUKAI, K., TOGURI, J. M., STUBINA, N. M., \& YOSHITOMI, J., A Mechanism for the local corrosion of Immersion Nozzles. ISIJ International, v. 29. (6), p. 1989.

REZENDE, R. V. P. Modelagem Matemática e Desenvolvimento de Metodologia Computacional para a simulação do Escoamento Bifásico de ar e Ferro-Gusa em Canal de Corrida de Alto-Forno. Dissertação (Mestrado). Engenharia Mecânica, Universidade federal de Santa Catarina, Florianópolis, 2008. xxx, 156 p.

SHESTOPALOV, I. I., DENISOV, A. V., MEL'NIKOV, P. N., MAKAROV, V. S., UNIGOVSKI, L. B., SHUL'MAN, V. G., PROKHOROV, V. N., VERTMAN, G. M., \& TSERA, I. A., Improving separation of melting productis in the main trough of a blast furnace. Metallurgy, v. 6. p. 32-33, 1988.

STEVENSON, P., \& HE, Q., Slug flow in a blast furnace taphole. Chemical Engineering and Processing, v. 44. (10), p. 1094-1097, 2005. 